\title{
A Cross-Sectional Analysis of the Association between Physical Activity and Visceral Adipose Tissue Accumulation in a Multiethnic Cohort
}

\author{
Iris A. Lesser, ${ }^{1}$ Ann C. Yew, ${ }^{1}$ Dawn C. Mackey, ${ }^{1,2}$ and Scott A. Lear ${ }^{1,3,4}$ \\ ${ }^{1}$ Department of Biomedical Physiology and Kinesiology, Simon Fraser University, Vancouver, BC, Canada V6B 5K3 \\ ${ }^{2}$ Centre for Hip Health and Mobility, Vancouver Coastal Health Research Institute, Vancouver, BC, Canada V5Z 1M9 \\ ${ }^{3}$ Faculty of Health Sciences, Simon Fraser University, Burnaby, BC, Canada V5A 1S6 \\ ${ }^{4}$ Division of Cardiology, Providence Health Care, Vancouver, BC, Canada V6Z 1 Y6 \\ Correspondence should be addressed to Scott A. Lear, slear@providencehealth.bc.ca
}

Received 6 July 2012; Revised 24 August 2012; Accepted 28 August 2012

Academic Editor: Bernhard H. Breier

Copyright ( 2012 Iris A. Lesser et al. This is an open access article distributed under the Creative Commons Attribution License, which permits unrestricted use, distribution, and reproduction in any medium, provided the original work is properly cited.

\begin{abstract}
Higher levels of VAT at the same body size and lower levels of physical activity (PA) have been reported in persons of Chinese and South Asian origin compared to European origin. The purpose of this study was to test the hypothesis that higher levels of VAT in persons of Chinese and South Asian origin versus European origin are associated with lower levels of PA. Chinese, European, and South Asian participants were assessed for sociodemographics, obesity-related measures, anthropometrics, and PA. Bivariate correlations, analysis of covariance, and regression models were used to explore ethnic differences in PA and the role of PA in explaining obesity-related measures. We observed ethnic differences in both body fat distribution and PA. Chinese and South Asians had higher amounts of VAT at a given BMI but lower amounts of moderate PA, vigorous PA, and moderate-to-vigorous PA (MVPA). Furthermore, we found ethnic-specific differences in the associations between body fat distribution and PA with only Europeans showing a consistent negative relationship between body fat distribution and PA. When ethnic differences in PA were taken into account, there were no longer any differences in VAT between the Chinese and European groups, while VAT remained higher in South Asians than Europeans.
\end{abstract}

\section{Introduction}

The prevalence of obesity and related chronic noncommunicable diseases is increasingly affecting the health of populations worldwide with the greatest increases in obesity occurring in countries such as China and India [1]. A growing body of research suggests that central distribution of body fat may be more important than general obesity in understanding differences in cardiovascular disease (CVD) risk factor profiles between individuals [2]. Even at desirable values of body mass index, elevated values of waist circumference are associated with increased risk for disease outcomes such as type 2 diabetes and CVD [3-5]. This is likely due to the fact that waist circumference is reflective of excess visceral adipose tissue (VAT), which has been shown to be highly associated with metabolic and CVD risk [6-8] and to be an independent predictor of all-cause mortality in men [9]. Previous research has shown that for a given waist circumference and body fat percentage, Chinese and South Asian people have a greater amount of VAT than their European counterparts [10], which may predispose these populations to increased risk for related diseases [11].

Physical activity (PA) has been proposed as one of the most effective therapies for reducing VAT [12]. Crosssectional studies of PA have shown associations between higher PA levels and lower VAT [13], and a number of PA intervention studies have demonstrated significant reductions in VAT $[12,14-16]$. PA-induced reductions in VAT have also been reported in the presence of only modest $(<3 \%)$, or even no weight loss $[14,17,18]$. A systematic review of aerobic PA and VAT found that as little as 10 MET hours per week (approximately 150 minutes per week 
of moderate PA such as brisk walking) is adequate for a significant reduction in VAT [19]; however, vigorousintensity PA may result in greater reductions in VAT than moderate-intensity PA [20, 21].

It has been suggested that changes in VAT in response to PA are heterogeneous [18], and the response to exercise may depend on differences in body fat distribution, such that individuals defined as "thin on the outside fat on the inside" (TOFI) may benefit more than others [22]. This subphenotype description (TOFI) is consistent with our earlier findings of body fat distribution in Chinese and South Asians [10]. However, the response of this subphenotype to PA has been poorly explored and requires further investigation.

Given current knowledge about VAT and low levels of physical activity (PA) in Chinese and South Asians compared to Europeans, [23] ethnic differences in PA may have a role in previously reported ethnic differences in VAT. We therefore hypothesized that ethnic differences in VAT are due, in part, to ethnic differences in PA which we assessed in both amount of PA and intensity of PA. We also explored the effect of ethnic differences in PA on differences in total body fat and subcutaneous abdominal adipose tissue (SAAT).

\section{Methods}

Study participants were 603 apparently healthy men and women between 30 and 65 years of age of Chinese $(n=207)$, European $(n=200)$, and South Asian $(n=196)$ origins who were recruited from Vancouver, Canada and enrolled in the multicultural health assessment trial. The M-CHAT study was designed to investigate body fat distribution in different ethnic groups and has been described in detail [24]. Briefly, study participants were recruited to ensure equal numbers of men and women in each ethnic group and across ranges of body mass index $\left(18.5-24.9,25-29.9\right.$, and $\left.\geq 30 \mathrm{~kg} / \mathrm{m}^{2}\right)$. Participants were excluded if they self-reported CVD and/or diabetes, had undergone recent weight change (greater than $2 \mathrm{~kg}$ in 3 months prior to assessment date), if they were on medication known to affect type 2 diabetes and CVD risk factors (lipid-lowering, antihypertensive, hypoglycemic, hormone replacement therapy, or insulin), or if they had significant prosthetics or amputations. As we had difficulty identifying an adequate sample of Chinese participants in the highest BMI range meeting our inclusion criteria, we changed our target for the upper range to a BMI of $\geq 28 \mathrm{~kg} / \mathrm{m}^{2}$. These changes to the sampling range resulted in a study cohort that although not evenly distributed across the BMI tertiles allowed for sufficient variation in BMI values within each ethnic group. The study protocol was approved by the Simon Fraser University Research Ethics Board, and all participants provided written informed consent.

2.1. Physical Activity Measurements. Physical activity was assessed using the Modifiable PA Questionnaire [25]. This questionnaire has previously been used with a multiethnic population inclusive of South Asians and Chinese to examine physical activity. The questionnaire gave participants a range of leisure time PA options to choose from, and they recalled how often and for how long they participated in each activity over a one-year time frame. Activities that were not listed were reported in the other category. Leisure-time PA included domestic activities such as housework, yard work, and shoveling as well as recreational and sporting activities such as walking, yoga, soccer, and swimming. Metabolic equivalents (METs) of reported activities were determined from the Ainsworth Compendium [26] and were categorized into moderate (3-6 METs) and vigorous ( $>6$ METs) intensities and reported as minutes per week. The sum of moderate-vigorous PA (MVPA) was calculated to reflect the total of moderate and vigorous minutes per week.

2.2. Body Composition Assessment. Abdominal adipose tissue was assessed by computerized tomography (CT) from a single cross-sectional CT scan centred at the L4/L5 intervertebral disc. All scans were performed with a CTi Advantage scanner (General Electric, Milwaukee, WI). Scan parameters were set at $120 \mathrm{kV}(\mathrm{p}), 300 \mathrm{~mA}$ for $1 \mathrm{~s}, 10 \mathrm{~mm}$ thickness, $512 \times 512$ matrix, using a $48 \mathrm{~cm}$ field of view. Computation of surface areas was conducted via the use of medical imaging software SliceOmatic v4.2 (TomoVision, Montreal, QC, Canada), using the attenuation range of $(-190)$ to $(-30)$ Hounsfield units for adipose tissue. VAT was calculated as total pixels and area within the attenuation range that fell within the visceral peritoneum (abdominal wall), and SAAT was calculated as the difference between total abdominal adipose tissue and VAT. Total body fat was assessed by dualenergy X-ray absorptiometry with a Norland XR-36 scanner (Norland Medical Systems, White Plains, NY).

2.3. Other Measurements. Participant age, sex, education, ethnicity, and smoking status were assessed by self-report. Weight in kilograms and height in metres were assessed with participants in light clothing (or hospital gowns), footwear removed, and pockets emptied. Body mass index was calculated as body mass in kilograms divided by height in metres squared. Waist circumference was recorded in centimetres as the average of two measures taken against the skin at the point of maximal narrowing from the anterior view following a normal expiration.

2.4. Statistical Analyses. All continuous variables were examined for normality, and non-normally distributed variables were transformed prior to analyses using the natural logarithm. To assess ethnic differences, we used chi-squared tests for categorical variables and one-way ANOVA with Bonferroni-adjusted post hoc tests for continuous variables. Pearson correlation coefficients were used to identify the linear association between moderate PA, vigorous PA, and MVPA, with VAT, SAAT, and total body fat, stratified by ethnicity and significant correlations, were used in the main regression analysis. Sequential linear regression models were constructed with ethnicity as the predictor variable and VAT, SAAT, or total body fat as the dependent variable, with adjustments for age, sex, BMI, smoking, and education. In model 1 ethnic differences in VAT were adjusted for age, sex, 
BMI, smoking, and education. To determine the influence of PA and the intensity of PA on the relationship between ethnicity and VAT, subsequent models were adjusted for the same covariates as model 1 , as well as either MVPA (model 2a) or both moderate and vigorous PA (model 2b). Significance was set at $P<0.05$ for main effects. All statistical analyses were performed with SPSS 17.0 software (SPSS, Chicago, IL).

\section{Results}

Participant age, education, and smoking status differed by ethnicity (Table 1). Mean BMI for the Chinese, European, and South Asian participants was 25.8, 27.8, and 27.9, respectively. There were significant ethnic differences in all of the body composition and physical activity measures. Of note, VAT was significantly higher in South Asian compared to European $(P=0.037)$ participants. SAAT differed between all ethnic groups with the lowest values in Chinese followed by Europeans $(P<0.001)$, and total body fat was significantly higher in South Asians compared to Europeans $(P<0.001)$ and in Europeans compared to Chinese $(P<$ 0.001). Chinese and South Asian participants had lower moderate and MVPA than Europeans $(P<0.001)$, while vigorous PA was also lower in Chinese compared to European participants $(P=0.004)$ (Table 1$)$.

After adjusting for age, sex, BMI, education, and smoking status, VAT was higher in both Chinese $(P=0.004)$ and South Asians $(P<0.001)$ than Europeans (Table 3, model 1). Further adjustment for MVPA did not substantially alter these associations (Table 3 model 2a). After we adjusted for both moderate PA and vigorous PA (Table 3 model 2b), the difference in VAT between Chinese and Europeans was no longer apparent $(P=0.109)$, while VAT remained significantly greater in South Asians compared to Europeans $(P<0.001)$. In this model, moderate PA and vigorous PA were both significantly associated with VAT $(P=0.013$, $P<0.001)$ and accounted for an additional $3.6 \%$ of the variance in VAT.

There were no differences in SAAT between Chinese and Europeans, but South Asians had greater amounts of SAAT than Europeans $(P<0.001)$ when adjusting for age, sex, BMI, education, and smoking status. Further adjustment for MVPA (Table 3, model 2a) or moderate PA and vigorous PA (Table 3, model 2b) had little impact on the ethnic differences. Vigorous PA was significantly associated with SAAT $(P=0.026)$, while moderate PA was not significantly associated with SAAT $(P=0.283)$, accounting for only an additional $0.4 \%$ of the variance in SAAT.

Total body fat was lower in Chinese $(P<0.001)$ and higher in South Asians $(P<0.001)$ compared to Europeans, adjusted for age, sex, BMI, education, and smoking status. Further adjustment for MVPA (Table 3, model 2a) or moderate PA and vigorous PA (Table 3 , model 2b) had little impact on the ethnic differences. Vigorous PA was significantly associated with total body fat $(P<0.001)$, while moderate PA was not significantly associated with body fat $(P=0.458)$, accounting for an additional $0.4 \%$ of the variance in total body fat.

\section{Discussion}

The purpose of this investigation was to assess ethnic differences in PA amount and intensity and to explore whether these PA differences may explain ethnic differences in total and centralized body fat. We found that in comparison to Europeans, both Chinese and South Asians had lower levels of moderate PA and MVPA and Chinese had lower levels of vigorous PA. These lower levels of PA were accompanied by higher adjusted amounts of VAT. Both levels of moderate PA and vigorous PA were negatively associated with VAT in Chinese and Europeans but only vigorous PA was negatively associated with VAT in South Asians. When ethnic differences in PA were taken into account, there were no longer any differences in VAT between the Chinese and European groups, while VAT remained higher in South Asians. Ethnic differences in PA did not influence ethnic differences in SAAT or total body fat.

Our findings of lower levels of PA in Chinese and South Asian groups than Europeans are consistent with what has been reported previously among ethnic minority populations in Canada $[23,27]$, the UK $[28,29]$, and the US [30]. Liu et al. reported that Chinese and South Asian men and women were 1.58 and 1.66 times, respectively, more likely to be physically inactive compared to Europeans [23]. High levels of physical inactivity in Chinese and South Asian populations have also been found to be independent risk factors for diabetes, which suggests that physical inactivity is an appropriate target for primary prevention efforts in these two high-risk populations [31].

It has previously been shown that vigorous intensity exercise significantly reduces VAT compared to moderate intensity exercise in primarily European populations [20, 32]. There is speculation that the greater response of VAT to vigorous intensity exercise may be due to the increased sensitivity of VAT to lipolytic stimulation during higher intensity exercise as increasing exercise intensity results in greater lipolysis which may facilitate greater postexercise energy expenditure and fat oxidation [33]. We found VAT to be significantly correlated with vigorous $\mathrm{PA}$ in all three ethnicities, which was not true for moderate PA.

While there is evidence that VAT is significantly reduced with vigorous intensity exercise, there is conflicting evidence regarding exercise intensity and SAAT reduction. We found no correlation between SAAT and PA in Chinese and South Asians while there was a significant correlation in Europeans with vigorous PA. Coker and colleagues did not observe a significant reduction in SAAT in either moderate or vigorous intensity exercise groups, while Irving et al. found a significant reduction in SAAT in their vigorous intensity exercise group but not their moderate intensity exercise group [20,32].

Vigorous exercise but not moderate exercise has been shown to reduce total body fat in European men and women $[20,34]$. We found total body fat to be significantly 


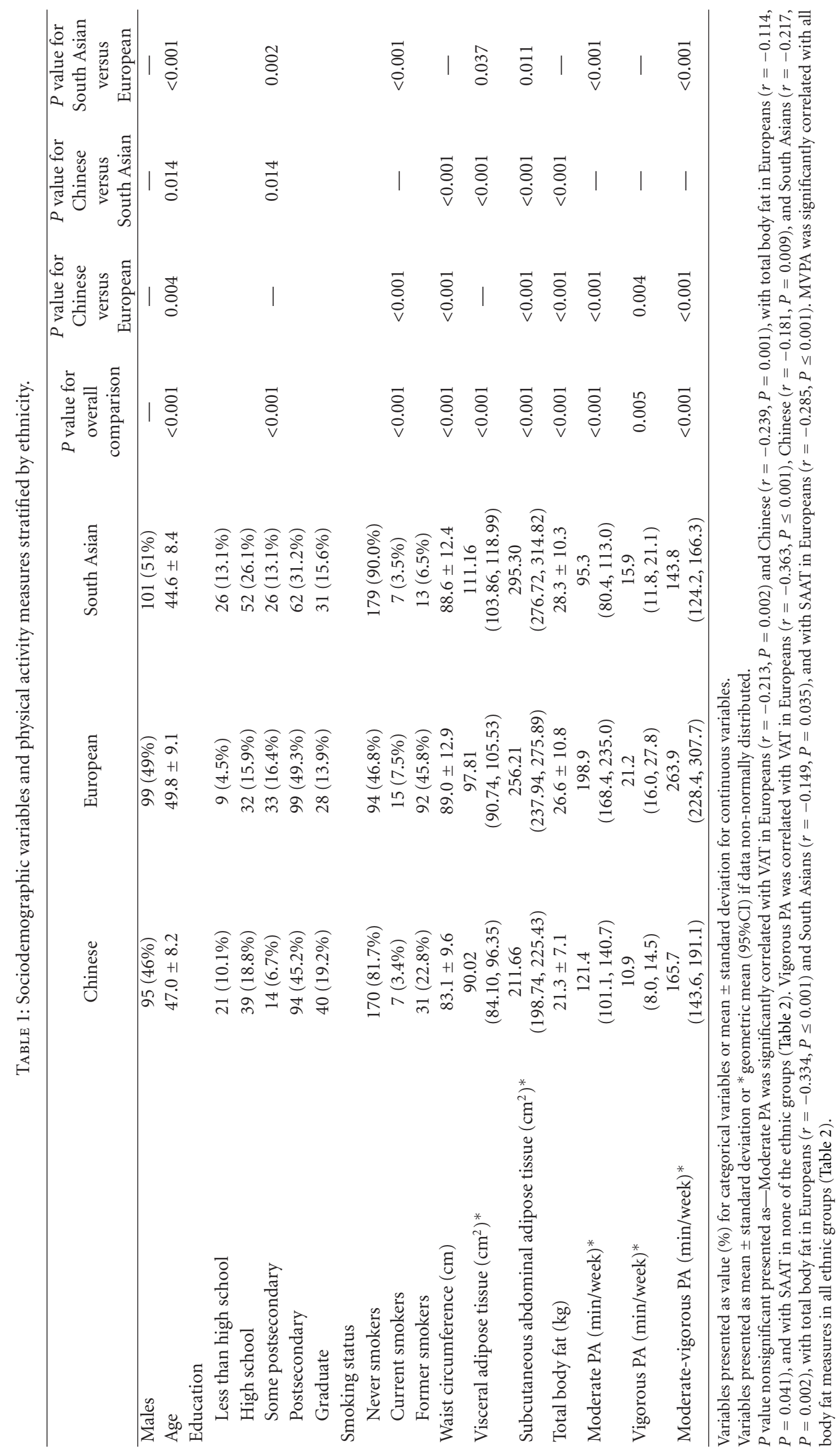


TABLE 2: Bivariate correlations between body fat distribution variables and moderate PA, vigorous PA, and MVPA across ethnicities.

\begin{tabular}{|c|c|c|c|c|c|c|c|c|c|}
\hline & \multicolumn{3}{|c|}{ Moderate PA_ln } & \multicolumn{3}{|c|}{ Vigorous PA_ln } & \multicolumn{3}{|c|}{ MVPA_ln } \\
\hline & Chinese & Europeans & South asian & Chinese & Europeans & South asian & Chinese & Europeans & South Asian \\
\hline VAT_ln & $-0.239 * *$ & $-0.213^{* *}$ & -0.082 & $-0.181^{* *}$ & $-0.363^{* * *}$ & $-0.217^{* *}$ & $-0.273^{* * *}$ & $-0.303^{* * *}$ & $-0.176^{*}$ \\
\hline SAAT_ln & -0.100 & -0.128 & -0.106 & -0.117 & $-0.285^{* * *}$ & -0.124 & $-0.143^{*}$ & $-0.225^{* *}$ & $-0.192^{* *}$ \\
\hline Total body fat (kg) & -0.091 & $-0.144^{*}$ & -0.064 & -0.136 & $-0.334^{* * *}$ & $-0.149^{*}$ & $-0.150^{*}$ & $-0.253^{* * *}$ & $-0.163^{*}$ \\
\hline
\end{tabular}

${ }^{*} P<0.05,{ }^{*} P<0.01, * * * P<0.001$.

VAT: visceral adipose tissue, SAAT: subcutaneous abdominal adipose tissue, PA: physical activity, MVPA: moderate-vigorous physical activity.

TABLE 3: Multiple linear regression analyses assessing physical activity as a predictor variable in body fat distribution.

\begin{tabular}{|c|c|c|c|c|c|c|}
\hline & \multicolumn{2}{|l|}{ VAT } & \multicolumn{2}{|c|}{ SAAT } & \multicolumn{2}{|c|}{ Total body fat } \\
\hline & Standardized beta & $P$ value & Standardized beta & $P$ value & Standardized beta & $P$ value \\
\hline \multicolumn{7}{|c|}{ Model 1} \\
\hline Chinese* & 0.105 & 0.004 & -0.038 & 0.178 & -0.091 & $<0.001$ \\
\hline South Asian* & 0.184 & $<0.001$ & 0.145 & $<0.001$ & 0.096 & $<0.001$ \\
\hline \multicolumn{7}{|c|}{ Model 2a } \\
\hline Chinese* & 0.073 & 0.041 & -0.049 & 0.089 & -0.101 & $<0.001$ \\
\hline South Asian* & 0.152 & $<0.001$ & 0.135 & $<0.001$ & 0.086 & $<0.001$ \\
\hline \multicolumn{7}{|c|}{ Model 2b } \\
\hline Chinese* & 0.054 & 0.109 & -0.054 & 0.065 & -0.109 & $<0.001$ \\
\hline South Asian* & 0.148 & $<0.001$ & 0.133 & $<0.001$ & 0.084 & $<0.001$ \\
\hline
\end{tabular}

* Compared to Europeans.

Model 1: adjusted for age, sex, education, BMI, former smoking, current smoking, and ethnicity.

Model 2a: model $1+$ MVPA.

Model 2b: model $1+$ Moderate PA + Vigorous PA.

correlated with moderate intensity PA in Europeans and with vigorous intensity PA in all ethnic groups. While differences between moderate and vigorous intensity exercise and body fat distribution were observed in the above studies, Slentz and colleagues found no significant difference in body fat distribution in a moderate or vigorous intensity exercise group of the same volume but found a significant difference with vigorous intensity exercise of a higher amount [35].

As we have reported earlier [10], VAT was higher in both Chinese and South Asian participants after taking into account key confounders such as BMI. When further adjusted by moderate PA and vigorous PA, there was no longer any difference in VAT between Chinese and Europeans, but the difference in VAT between South Asians and Europeans remained. This finding suggests that different mechanisms may explain ethnic-specific differences in VAT accumulation. For example, the higher VAT levels in Chinese compared to Europeans may be mediated by lower levels of PA among Chinese, whereas higher VAT levels in South Asians compared to Europeans do not appear to be related to lower levels of PA. Indeed, Ross and Bradshaw have indicated that changes in VAT in response to exercise are heterogeneous, and some individuals may benefit more from exercise than others [18].

Despite taking into account their lower levels of PA, South Asians still had higher levels of VAT than the Europeans. In recent years, a number of hypotheses have been presented that may explain the South Asian obesity phenotype of elevated VAT and total body fat. This includes the development of dysfunctional adipocytes through intrauterine origins (including epigenetics) and a lower threshold for storing adipose tissue in subcutaneous stores [36, 37]. It has also been theorized that there may be genetic or epigenetic factors which influence catecholamine-induced lipolysis during exercise [38] which may in turn differentiate the response of VAT to exercise in the South Asian population. However, it is important to note that the South Asian population studied here reported very low levels of PA, and it is possible that a higher volume or intensity of PA may alter this subphenotype.

Interestingly, when MVPA was added to the model there remained a difference in VAT between both ethnic groups and Europeans. However, VAT was significantly correlated with both PA intensities in our sample, therefore, to determine which intensity was associated with the ethnic differences in VAT, each intensity was added independently into the model. By taking into account moderate PA as an independent variable, there was no longer an ethnic difference in VAT between Chinese and Europeans. A doseresponse relationship with vigorous intensity exercise and VAT, SAAT, and total body fat has previously been observed, and it was suggested that total energy expenditure may be a more important predictor than intensity for fat mobilization [35]. We found median minutes per week for vigorous PA to be 10.9, 40.5, and 22.8 for Chinese, Europeans, and South Asians, respectively, while moderate PA was reported as 139.2, 230.8, and 110.2 minutes per week, respectively. This volume of PA for Chinese and South Asians is lower than the 
150 minutes per week which has previously been reported as the amount necessary to significantly reduce VAT [19].

This study has certain limitations. As it was crosssectional, it is limited in its ability to infer causality. While there is evidence to suggest that obese individuals may be limited in the amount of PA, they can engage in as a result of their obesity [39], the overwhelming majority of our participants had a BMI well below the levels at which PA is likely to be limited by body size (BMI of 25.8, 27.8, and 27.9 for Chinese, European and South Asian resp.), and therefore we believe that in our population, the act of PA preceded that of body fat accumulation. The self-report of a oneyear recall of PA is another limitation, as individuals tend to overestimate PA due to social desirability bias [40]. Although this is a significant limitation in PA research, the self-report bias is likely similar across ethnic groups. In support of this notion, the physical activity questionnaire used in this study has previously been used in a different multiethnic cohort of South Asians and Chinese to define PA [25]. Also, the lower levels of PA reported in South Asians and Chinese compared to Europeans are consistent with previous observations in these populations [23]. Nevertheless, it is likely that individuals in our population sample overreported PA participation. Classification of PA into intensity using the Ainsworth Compendium may also have resulted in error if the intensity of the activity reported differed from what is indicated in the Compendium which may influence the results if PA was categorized as moderate when it should have been vigorous or vice versa. We must also acknowledge the limitations of using the thresholds for MPA and VPA of 3 and $6 \mathrm{METs}$, respectively. While these absolute cutoffs have been used in various PA studies [41, 42], they may not fully capture the relative terms of "moderate" and "vigorous" in that 6 METs in a highly trained individual may only illicit a moderate response and similarly, in an older, more frail person, 3 METs may be considered vigorous. In our study, however, given the narrow range of age and abilities, this limitation is not likely to be as prominent in a more heterogeneous population. Lastly, our study cohort was purposely recruited from a wide range of BMI values and not a random population sample. However, this design allowed us to investigate these associations across a range of body fat and VAT amounts as opposed to a narrower range of body sizes one might expect in these different ethnic groups.

The strengths of this study are the use of gold standard techniques for measurement of body composition: computed tomography for VAT and SAAT and dual energy X-ray absorptiometry for total body fat. Additionally, the population sampled was purposely recruited based on ethnicity and BMI.

\section{Conclusions}

In conclusion, in this cross-sectional study, we observed ethnic differences in both body fat distribution and PA; Chinese and South Asians had higher amounts of VAT at a given BMI but lower amounts of moderate PA, vigorous PA, and MVPA than Europeans. Furthermore, we found ethnicspecific differences in the associations between body fat distribution and PA with only Europeans showing a consistent linear relationship between body fat distribution and PA in correlational analyses. When ethnic differences in PA were taken into account, there were no longer any differences in VAT between the Chinese and European groups, while VAT remained higher in South Asians. Encouraging PA in ethnic minority populations is essential as these populations are growing in Canada. An exercise intervention study is necessary to further elucidate the effects of PA on body fat distribution in ethnic populations.

\section{Abbreviations}

PA: Physical activity

MVPA: Moderate-vigorous physical activity

VAT: Visceral adipose tissue

SAAT: Subcutaneous abdominal adipose tissue.

\section{Conflict of Interests}

The authors declare no conflict of interests.

\section{Authors' Contribution}

I. A. Lesser drafted the paper, performed the statistical analysis, and conceived of the study. A. C. Yew participated in the design of the study and conceived of the study. D. C. Mackey participated in interpretation of the data and revised the paper for important intellectual content. S. A. Lear conducted data collection, participated in the design of the study, and drafted the paper. All authors read and approved the final paper.

\section{Acknowledgments}

This work was supported by the Canadian Institutes of Health Research and the Institute of Nutrition, Metabolism, and Diabetes. SAL is a Canadian Institutes of Health Research New Investigator and holds the Pfizer/Heart \& Stroke Foundation Chair in cardiovascular prevention research at St. Paul's Hospital. The authors had complete independence from the funders.

\section{References}

[1] World Health Organization, The Global Burden of Disease: 2004, 2008.

[2] J. P. Despres, S. Moorjani, P. J. Lupien, A. Tremblay, A. Nadeau, and C. Bouchard, "Regional distribution of body fat, plasma lipoproteins, and cardiovascular disease," Arteriosclerosis, vol. 10, no. 4, pp. 497-511, 1990.

[3] S. Yusuf, S. Hawken, S. Ônpuu et al., "Obesity and the risk of myocardial infarction in 27, 000 participants from 52 countries: a case-control study," The Lancet, vol. 366, no. 9497, pp. 1640-1649, 2005.

[4] C. Zhang, K. M. Rexrode, R. M. van Dam, T. Y. Li, and F. B. Hu, "Abdominal obesity and the risk of all-cause, 
cardiovascular, and cancer mortality: sixteen years of followup in US women," Circulation, vol. 117, no. 13, pp. 1658-1667, 2008.

[5] K. M. Rexrode, V. J. Carey, C. H. Hennekens et al., "Abdominal adiposity and coronary heart disease in women," Journal of the American Medical Association, vol. 280, no. 21, pp. 1843-1848, 1998.

[6] J. P. Despres, M. C. Pouliot, S. Moorjani et al., "Loss of abdominal fat and metabolic response to exercise training in obese women," American Journal of Physiology, vol. 261, no. 2, pp. E159-E167, 1991.

[7] J. P. Després, I. Lemieux, and D. Prud'homme, “Treatment of obesity: need to focus on high risk abdominally obese patients," British Medical Journal, vol. 322, no. 7288, pp. 716720, 2001.

[8] J. P. Després, B. J. Arsenault, M. Côté, A. Cartier, and I. Lemieux, "Abdominal obesity: the cholesterol of the 21st century?" The Canadian Journal of Cardiology, vol. 24, supplement D, pp. 7D-12D, 2008.

[9] J. L. Kuk, P. T. Katzmarzyk, M. Z. Nichaman, T. S. Church, S. N. Blair, and R. Ross, "Visceral fat is an independent predictor of all-cause mortality in men," Obesity, vol. 14, no. 2, pp. 336$341,2006$.

[10] S. A. Lear, K. H. Humphries, S. Kohli, A. Chockalingam, J. J. Frohlich, and C. L. Birmingham, "Visceral adipose tissue accumulation differs according to ethnic background: results of the Multicultural Community Health Assessment Trial (MCHAT)," American Journal of Clinical Nutrition, vol. 86, no. 2, pp. 353-359, 2007.

[11] S. A. Lear, A. Chockalingam, S. Kohli, C. G. Richardson, and K. H. Humphries, "Elevation in cardiovascular disease risk in south asians is mediated by differences in visceral adipose tissue," Obesity, vol. 20, no. 6, pp. 1293-1300, 2012.

[12] R. Ross, I. Janssen, J. Dawson et al., "Exercise-induced reduction in obesity and insulin resistance in women: a randomized controlled trial," Obesity Research, vol. 12, no. 5, pp. 789798, 2004.

[13] E. A. Molenaar, J. M. Massaro, P. F. Jacques et al., "Association of lifestyle factors with abdominal subcutaneous and visceral adiposity the framingham heart study," Diabetes Care, vol. 32, no. 3, pp. 505-510, 2009.

[14] R. Ross, J. A. Freeman, and I. Janssen, "Exercise alone is an effective strategy for reducing obesity and related comorbidities," Exercise and Sport Sciences Reviews, vol. 28, no. 4, pp. 165-170, 2000.

[15] I. Giannopoulou, L. L. Ploutz-Snyder, R. Carhart et al., "Exercise is required for visceral fat loss in postmenopausal women with type 2 diabetes," Journal of Clinical Endocrinology and Metabolism, vol. 90, no. 3, pp. 1511-1518, 2005.

[16] V. B. O'Leary, C. M. Marchetti, R. K. Krishnan, B. P. Stetzer, F. Gonzalez, and J. P. Kirwan, "Exercise-induced reversal of insulin resistance in obese elderly is associated with reduced visceral fat," Journal of Applied Physiology, vol. 100, no. 5, pp. 1584-1589, 2006.

[17] S. Lee, J. L. Kuk, L. E. Davidson et al., "Exercise without weight loss is an effective strategy for obesity reduction in obese individuals with and without Type 2 diabetes," Journal of Applied Physiology, vol. 99, no. 3, pp. 1220-1225, 2005.

[18] R. Ross and A. J. Bradshaw, "The future of obesity reduction: beyond weight loss," Nature Reviews Endocrinology, vol. 5, no. 6, pp. 319-325, 2009.

[19] K. Ohkawara, S. Tanaka, M. Miyachi, K. Ishikawa-Takata, and I. Tabata, "A dose-response relation between aerobic exercise and visceral fat reduction: systematic review of clinical trials,"
International Journal of Obesity, vol. 31, no. 12, pp. 1786-1797, 2007.

[20] B. A. Irving, C. K. Davis, D. W. Brock et al., "Effect of exercise training intensity on abdominal visceral fat and body composition," Medicine and Science in Sports and Exercise, vol. 40, no. 11, pp. 1863-1872, 2008.

[21] H. Sasai, Y. Katayama, Y. Nakata et al., "The effects of vigorous physical activity on intra-abdominal fat levels: a preliminary study of middle-aged Japanese men," Diabetes Research and Clinical Practice, vol. 88, no. 1, pp. 34-41, 2010.

[22] E. L. Thomas et al., "The missing risk: MRI and MRS phenotyping of abdominal adiposity and ectopic fat," Obesity, vol. 20, pp. 76-87, 2012.

[23] R. Liu, L. So, S. Mohan, N. Khan, K. King, and H. Quan, "Cardiovascular risk factors in ethnic populations within Canada: results from national cross-sectional surveys," Open Medicine, vol. 4, no. 3, pp. E143-E153, 2010.

[24] S. A. Lear, C. L. Birmingham, A. Chockalingam, and K. H. Humphries, "Study design of the multicultural community health assessment trial (M-CHAT): a comparison of body fat distribution in four distinct populations," Ethnicity and Disease, vol. 16, no. 1, pp. 96-100, 2006.

[25] M. A. Pereira, A. M. Kriska, M. L. Joswiak et al., "Physical inactivity and glucose intolerance in the multiethnic island of Mauritius," Medicine and Science in Sports and Exercise, vol. 27, no. 12, pp. 1626-1634, 1995.

[26] B. E. Ainsworth, W. L. Haskell, S. D. Herrmann et al., "2011 compendium of physical activities: a second update of codes and MET values," Medicine and Science in Sports and Exercise, vol. 43, no. 8, pp. 1575-1581, 2011.

[27] S. Dogra, B. A. Meisner, and C. I. Ardern, "Variation in mode of physical activity by ethnicity and time since immigration: a cross-sectional analysis," International Journal of Behavioral Nutrition and Physical Activity, vol. 7, article 75, 2010.

[28] P. Zaninotto, J. Mindell, and V. Hirani, "Prevalence of cardiovascular risk factors among ethnic groups: results from the Health Surveys for England," Atherosclerosis, vol. 195, no. 1, pp. e48-e57, 2007.

[29] L. Hayes, M. White, N. Unwin et al., "Patterns of physical activity and relationship with risk markers for cardiovascular disease and diabetes in Indian, Pakistani, Bangladeshi and European adults in a UK population," Journal of Public Health Medicine, vol. 24, no. 3, pp. 170-178, 2002.

[30] J. Ye, G. Rust, P. Baltrus, and E. Daniels, "Cardiovascular risk factors among Asian Americans: results from a National Health Survey," Annals of Epidemiology, vol. 19, no. 10, pp. 718-723, 2009.

[31] G. K. Dowse, P. Z. Zimmet, H. Gareeboo et al., "Abdominal obesity and physical inactivity as risk factors for NIDDM and impaired glucose tolerance in Indian, Creole, and Chinese Mauritians," Diabetes Care, vol. 14, no. 4, pp. 271-282, 1991.

[32] R. H. Coker, R. H. Williams, P. M. Kortebein, D. H. Sullivan, and W. J. Evans, "Influence of exercise intensity on abdominal fat and adiponectin in elderly adults," Metabolic Syndrome and Related Disorders, vol. 7, no. 4, pp. 363-368, 2009.

[33] C. J. Pritzlaff, L. Wideman, J. Blumer et al., "Catecholamine release, growth hormone secretion, and energy expenditure during exercise vs. recovery in men," Journal of Applied Physiology, vol. 89, no. 3, pp. 937-946, 2000.

[34] A. Tremblay, J. P. Despres, C. Leblanc et al., "Effect of intensity of physical activity on body fatness and fat distribution," American Journal of Clinical Nutrition, vol. 51, no. 2, pp. 153157, 1990. 
[35] C. A. Slentz, B. D. Duscha, J. L. Johnson et al., "Effects of the amount of exercise on body weight, body composition, and measures of central obesity: STRRIDE-a Randomized Controlled Study," Archives of Internal Medicine, vol. 164, no. 1, pp. 31-39, 2004.

[36] A. D. Sniderman, R. Bhopal, D. Prabhakaran, N. Sarrafzadegan, and A. Tchernof, "Why might South Asians be so susceptible to central obesity and its atherogenic consequences? The adipose tissue overflow hypothesis," International Journal of Epidemiology, vol. 36, no. 1, pp. 220-225, 2007.

[37] C. S. Yajnik, "The lifecycle effects of nutrition and body size on adult adiposity, diabetes and cardiovascular disease," Obesity Reviews, vol. 3, no. 3, pp. 217-224, 2002.

[38] P. Arner, "Impact of exercise on adipose tissue metabolism in humans," International Journal of Obesity, vol. 19, no. 4, pp. S18-S21, 1995.

[39] I. Leroux, C. E. Dionne, R. Bourbonnais, and C. Brisson, "Prevalence of musculoskeletal activity limitation and associated factors among adults in the general population in the 1998 Quebec Health Survey," Journal of Rheumatology, vol. 32, no. 9, pp. 1794-1804, 2005.

[40] S. A. Adams, C. E. Matthews, and C. B. Ebbeling, "The effect of social desirability and social approval on self-reports of physical activity," American Journal of Epidemiology, vol. 161, no. 9, article 899, 2005.

[41] M. L. Irwin, E. J. Mayer-Davis, C. L. Addy et al., "Moderateintensity physical activity and fasting insulin levels in women: the Cross-Cultural Activity Participation Study," Diabetes Care, vol. 23, no. 4, pp. 449-454, 2000.

[42] S. Wilcox, M. L. Irwin, C. Addy et al., "Agreement between participant-rated and compendium-coded intensity of daily activities in a triethnic sample of women ages 40 years and older," Annals of Behavioral Medicine, vol. 23, no. 4, pp. 253 262, 2001. 


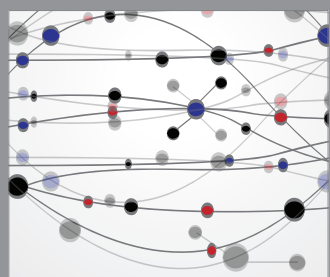

The Scientific World Journal
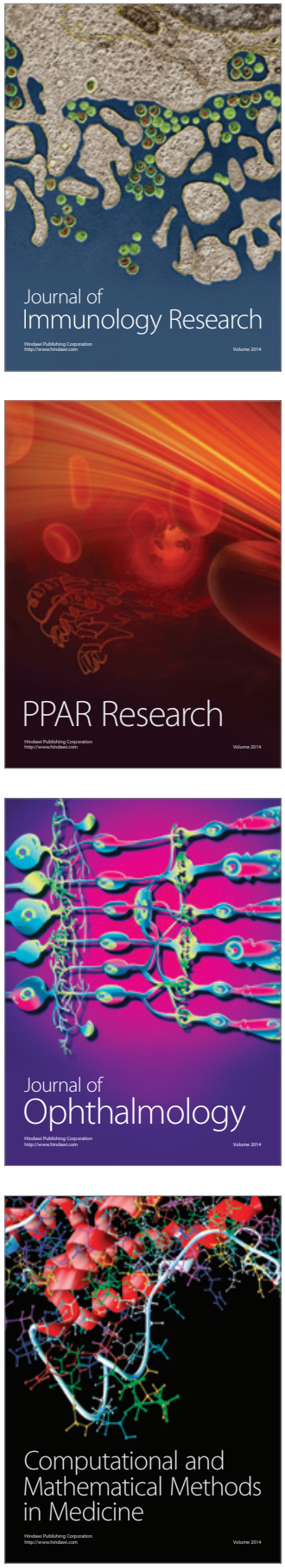

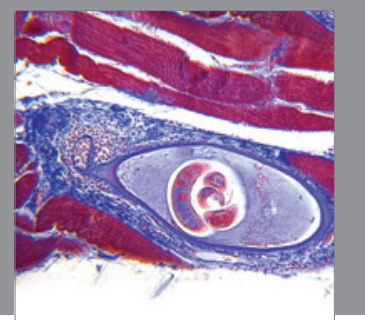

Gastroenterology

Research and Practice
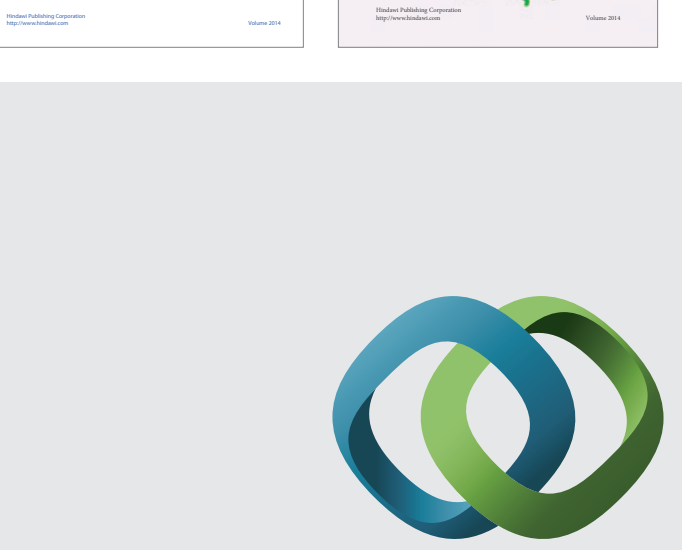

\section{Hindawi}

Submit your manuscripts at

http://www.hindawi.com
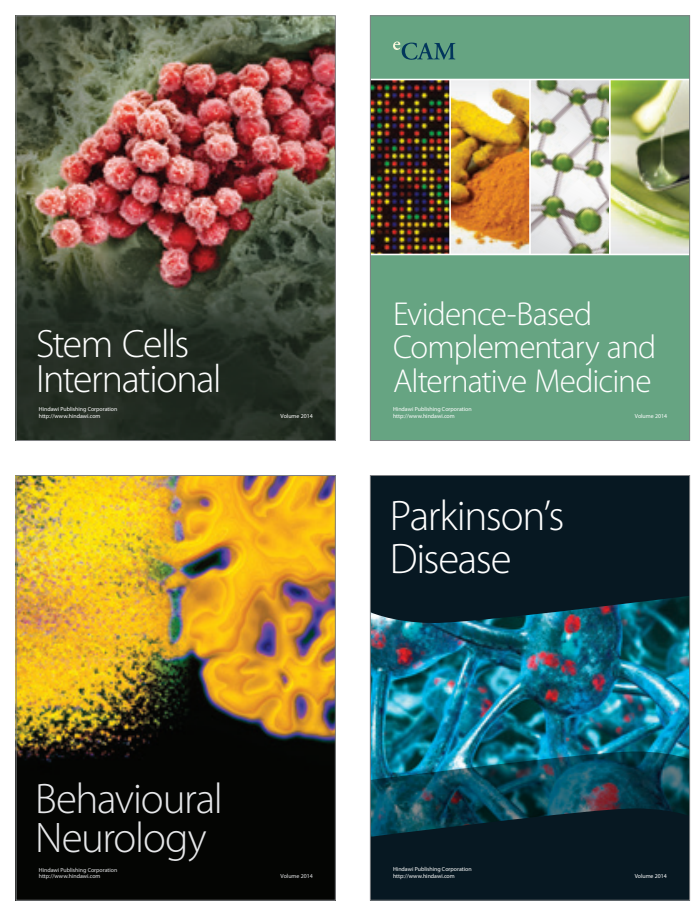

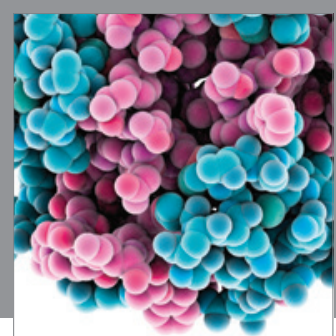

Journal of
Diabetes Research

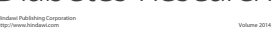

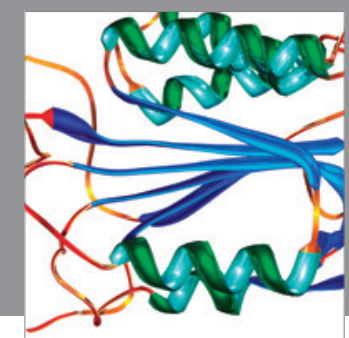

Disease Markers
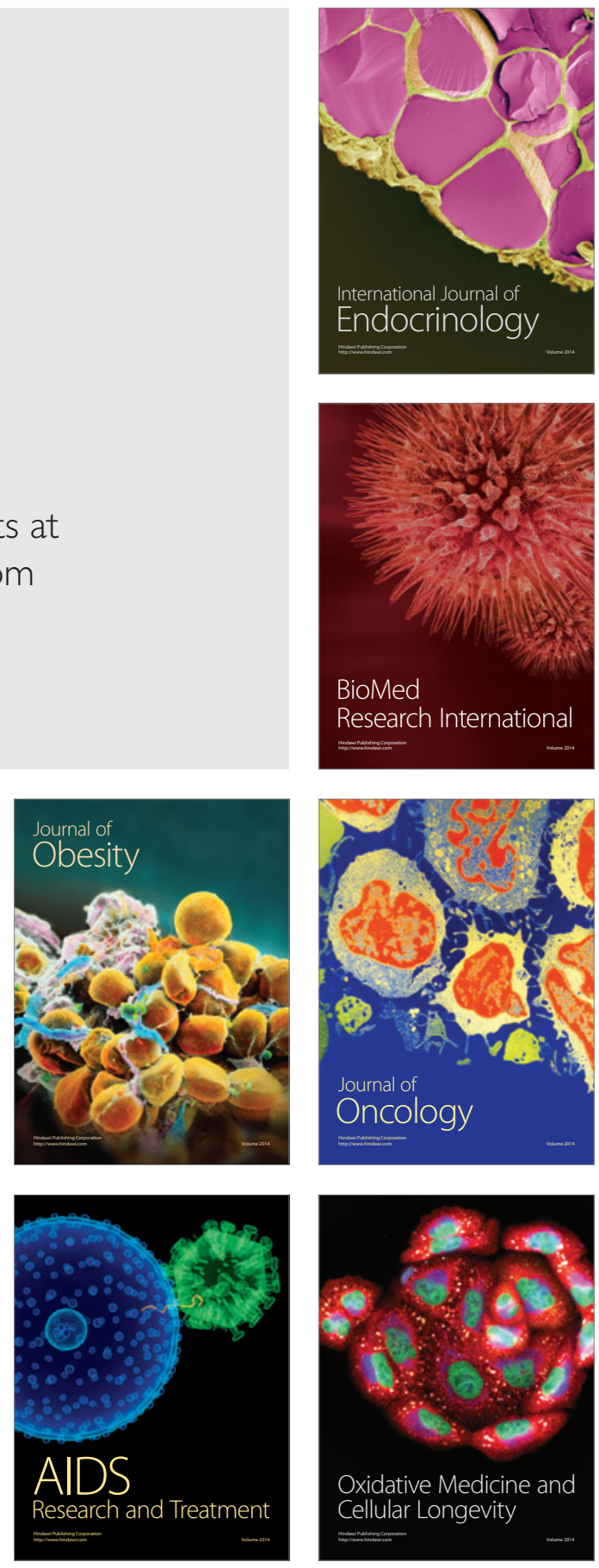